Research Article

\title{
Temperature Effects on Dynamic Properties of Suspended Cables Subjected to Dual Harmonic Excitations
}

\author{
Yaobing Zhao $\mathbb{D},{ }^{1,2}$ Panpan Zheng, ${ }^{1}$ Henghui Lin, ${ }^{1}$ and Chaohui Huang ${ }^{1}$ \\ ${ }^{1}$ College of Civil Engineering, Huaqiao University, Xiamen, Fujian 361021, China \\ ${ }^{2}$ Key Laboratory of Intelligent Infrastructure and Monitoring of Fujian Province, Huaqiao University, Xiamen, \\ Fujian 361021, China \\ Correspondence should be addressed to Yaobing Zhao; ybzhao@hqu.edu.cn
}

Received 8 September 2020; Revised 28 February 2021; Accepted 4 March 2021; Published 24 March 2021

Academic Editor: Pedro Museros

Copyright (c) 2021 Yaobing Zhao et al. This is an open access article distributed under the Creative Commons Attribution License, which permits unrestricted use, distribution, and reproduction in any medium, provided the original work is properly cited.

\begin{abstract}
The paper aims at studying the influences of temperature on the suspended cables' dynamical behaviors subjected to dual harmonic excitations in thermal environments. Significantly, the quadratic nonlinearity and the corresponding secondary resonances are considered. By introducing a tension variation factor, the nonlinear vibration equations of motion could be obtained based on the condensation model. By using Galerkin's procedure, the continuous model of the nonlinear system is reduced to a set of infinite models with quadratic and cubic nonlinearities. By using the multiple scales method, the resultant reduced model is solved and the stability analysis is also presented in two simultaneous resonance cases. Nonlinear dynamical behaviors with thermal effects are presented using bifurcation diagrams, time-history curves, phase portraits, frequency spectrums, and Poincaré sections. The numerical results show that thermal effects induce different scenarios. The sensitivities of linear (natural frequency) and nonlinear (quadratic and cubic) coefficients to temperature variations are different. The temperature may increase or decrease the response amplitudes depending on the excitation amplitude and the sag-to-span ratio. The inflection point is shifted and exhibited at a smaller or larger excitation amplitude in thermal environments. The resonant range between two Pitchfork bifurcations seems to be reduced when the temperature is decreasing. The response amplitude is very sensitive to temperature, and even an opposite spring behavior may be exhibited due to warming/cooling conditions. However, the periodic motions seem independent of temperature variations.
\end{abstract}

\section{Introduction}

With the increased significance of multiphysics phenomena occurring in many technological contexts, linear and nonlinear dynamic responses of materials and structures in thermal environments become a research topic of significant interest recently $[1,2]$. Temperature becomes a significant factor that may change the dynamic characteristics and responses of some essential structure elements, for example, beam [3], shells [4], pipes [5], and cables [6].

In the past few years, structures with flexible cables have attracted significant attention. The potential application of the fundamental research in practical industries is exhibited, especially the shape-finding [7] and dynamic analysis [8] as well as sliding mode control [9] of pantograph-catenary in high-speed railway engineering. These flexible systems especially possess low structural damping and small stiffness, and thus any disturbances may cause large-amplitude vibrations [10]. Therefore, a great deal of research has been conducted on the mathematical modeling and linear and nonlinear vibration analyses $[11,12]$.

Long-span cables experience a significant changing thermal environment during the construction and operation stages. The resultant temperature induces structural deformations that may affect the dynamical behaviors of the nonlinear system. Hence, it is necessary to investigate the linear and nonlinear vibration characteristics of cables after significant deformation induced by temperature effects [13-17]. To be more specific, the static analysis of a cable in thermal environments was presented in Irvine's monograph 
[18], and Treyssède [19] extended to a new thermal-stressed configuration. Then, a nonlinear dynamic model of the horizontal and inclined cables was established by Lepidi and Gattulli [20]. Furthermore, to explore the suspended cable's nonlinear vibration behaviors in thermal environments more fully and deeply, Zhao et al. presented some studies on the nonlinear primary [21] and secondary [22] resonant responses of suspended cables. Then, the temperature-dependent materials were illustrated in [23]. The influences of temperature on suspended cables' nonlinear vibration behaviors subjected to multifrequency excitations were also discussed by Zhao et al. [24].

On the other hand, considering different types of excitations, for example, direct/indirect excitation and auto-/ parametric excitation, the loads applied on structures are very complicated and always contain multifrequency harmonic forces. The rich and complex dynamics of nonlinear oscillations attracted the attention of the research community, leading to exploring the possibility of understanding dynamical behaviors in nonlinear systems subjected to multifrequency excitations [25-39].

Specifically, Ding and $\mathrm{Zu}$ [25] presented a study in periodic and chaotic responses of an axially accelerating viscoelastic beam subject to two-frequency excitations. Breslavsky and Amabili [26] investigated the nonlinear vibration of a water-filled, thin circular cylindrical shell, simply supported at the edges, to multiple harmonic excitations. Bauomy and EL-Sayed [27] studied the vibration behaviors of a vertical shaking conveyor subjected to multiple harmonic excitations. A Duffing system with multifrequency slow parametric excitations was investigated to report two novel bursting patterns by Han et al. [28]. Based on recent experimental observations, an inclined marine riser may experience multifrequency vortex-induced vibrations, and Alfosail and Younis [29] investigated the nonlinear motions considering the internal resonances. Briend et al. [30] analyzed the dynamics of an on-board rotor mounted on hydrodynamic finite-length bearings, excited by multifrequency parametric excitations induced by support motions. Ghadiri et al. [31] presented a study on nonlinear vibrations of single-layer graphene sheets resting on a visco-Pasternak foundation subjected to multifrequency excitation and a thermomagnetic field. Kalita and Dwivedy [32] presented a study on the nonlinear dynamics of a pneumatic artificial muscle under simultaneous simple and principle parametric resonance conditions. The analytical solutions and vibration behaviors of a cable-stayed bridge and a cable-stayed cantilever beam subjected to two external harmonic excitations were obtained [33, 39]. Xu et al. [34] investigated the vibration behaviors of multiparametric excitations and multifrequency external excitations of the rolling mill under entry thickness fluctuation of the strip. Ren et al. [35] investigated the amplitude-information-frequency characteristics for an underwater active electrolocation composition system subjected to multifrequency excitations. Jabar et al. [36] analytically and experimentally investigated the nonlinear oscillations of a clamped-clamped microbeam under multifrequency excitations. Tzanov et al. [37] studied the nonlinear resonant behaviors of a Si fractal NEMS resonator subjected to multifrequency excitations. Considering a piezoelectric nanoplate resting on a viscoelastic foundation, Ebrahimi and Hosseini [38] investigated its nonlinear vibration behaviors subjected to dual harmonic external excitation and thermoelectromechanical loads.

Furthermore, vibration absorber and control considering the multifrequency vibrations are also investigated. Pai et al. [40] presented a study on a new acoustic metamaterial beam consisting of a uniform isotropic beam and small twomass spring-mass-damper subsystems and acting as a multifrequency absorber. As to a nonlinear system consisting of a cantilever beam with a tip mass, Rezaei et al. [41] analyze the efficient energy harvesting from nonlinear oscillations of PZT beam excited by two simultaneous hard base accelerations. Zheng et al. [42] designed a multifrequency adaptive disturbance cancellation controller for a 6DOF microvibration excitation system. An active control strategy is applied by Sayed et al. [43] in the nonlinear vibrations of a buckled beam. Many simultaneous resonant cases have been investigated, and the worst case is the simultaneous primary and principal parametric in the existence of internal resonance cases.

Whether in practical applications or in laboratory experiments, the suspended cable is always subjected to multifrequency excitations and thermal loads. Hence, understanding the cables' dynamical behaviors considering multifrequency excitations and thermal effects is essential, since the temperature can affect the linear and nonlinear vibration characteristics. It is usually conducted to provide crucial information for damage evaluation and condition assessment. The suspended cable is a classical flexible nonlinear system with quadratic and cubic nonlinearities simultaneously. The initial curvature induces the quadratic nonlinearity, and a new thermal-stressed configuration will be generated in thermal environments. As to a string with a large initial tension force, only the pure cubic nonlinearity is considered. With the decrease in cable tension force, the effect of mixed quadratic and cubic nonlinearities should be considered due to the static configuration's change. In some circumstances, the quadratic nonlinearity may even overcome the cubic one and dominates the vibration behaviors. Besides, it is found out that the sensitivity of quadratic and cubic nonlinearity coefficients to temperature changes is different, and it depends on the cable tension force or the sag-to-span ratio.

In the present work, we still focus our attention on the nonlinear forced vibration response of suspended cables in thermal environments. In particular, we concentrate on the quadratic nonlinearity due to the curvature of the nonlinear system. To the best of the authors' knowledge, considering the primary, the second-order superharmonic, and the onehalf-order subharmonic resonances simultaneously, no previous study regarding the nonlinear dynamic behaviors of suspended cables with thermal effects has been reported before. The introduction of the research background and the literature review are illustrated in Section 1. The mathematical model of suspended cable excited by dual harmonic forces in thermal environments is described in Section 2. The 
perturbation procedures, as well as the stability analysis, are presented in Section 3. Section 4 gives some numerical examples and discussions about the temperature effects on vibration behaviors. A conclusion closes the whole work in Section 5.

\section{Mathematical Modeling}

A Cartesian coordinate system $O-x y$ is shown here. The nonlinear flexible system under consideration shown in Figure 1 contains a uniform horizontal cable of length $L$, initial sag $b$, thermal-stressed sag $b_{\Delta T}$, Young's modulus $E$, cross-sectional area $A$, and mass per length $m$. The static configuration is the original one without thermal effects. Besides, an extra new static configuration, a thermalstressed one, is generated due to the cables' expanding or contracting in thermal environments. Therefore, all the tension force, sag, and cable length are closely related to temperature variations. Under the external excitations, the displacements along two directions are expressed as $u(x, t)$ and $v(x, t)$, respectively. Dual distributed harmonic excitation forces along the cable length are applied along the suspended cable.

Generally, a suspended cable in thermal environments is affected by temperature changes in many resources, for example, tension forces (sag-to-span ratios), material properties, and boundary conditions. Here, material properties are assumed to be independent of temperature variations, and the cross-sectional area remains plane and uniform in thermal environments. Hence, by using the direct force method, a cubic equation including the cable tension force is obtained $[18,19]$.

$$
\Delta h^{3}+\left(2+\theta_{\Delta T}+\frac{\lambda^{2}}{24}\right) \Delta h^{2}+\left(1+2 \theta_{\Delta T}+\frac{\lambda^{2}}{12}\right) \Delta h+\theta_{\Delta T}=0
$$

where

$$
\begin{aligned}
\Delta h & =\frac{h}{H}, \\
\theta_{\Delta T} & =\frac{\alpha \Delta T L_{t}}{H L_{e}} E A, \\
\lambda^{2} & =\left(\frac{m g L}{H}\right)^{2} \frac{E A}{H}, \\
L_{t} & =L\left(1+\frac{m^{2} g^{2} L^{2}}{8 H^{2}}\right), \\
L_{e} & =L\left(1+\frac{m^{2} g^{2} L^{2}}{12 H^{2}}\right),
\end{aligned}
$$

where $h$ is an increment/reduction of the tension force induced by temperature changes; $H$ is the initial horizontal tension force; $\alpha$ is the thermal expansion coefficient; $m$ is the mass per unit length; $A, E$, and $\lambda^{2}$ are the cross-sectional area, Young's modulus, and Irvine parameter, respectively.
$\Delta T=T-T_{0}$, where $T_{0}$ is the initial temperature and $\Delta T$ denotes the temperature variation before loading.

Hence, the variation of the tension force and sag could be described by the following nondimensional parameter [20]:

$$
\chi_{\Delta T}^{2}=1+\Delta h=\frac{h+H}{H}=\frac{H_{\Delta T}}{H}=\frac{b}{b_{\Delta T}},
$$

where $b_{\Delta T}(b)$ and $H_{\Delta T}(H)$ are the cable sag and tension force with (without) thermal effects, respectively.

Neglecting temperature effects and adopting a kinematic condensation procedure, the partial differential equations of the suspended cable's planar motions could be obtained [11]. Moreover, an analytical model, including the horizontal suspended and inclined cables, incorporating the temperature effects, has been built by Lepidi and Gattulli [20]. Based on the obtained condensed mathematical model, the following continuous expressions for the in-plane displacements are obtained when considering thermal effects.

$$
\begin{aligned}
& m v_{t t}+c v_{t}-\chi_{\Delta T}^{2} H v_{x x}-\frac{E A}{L}\left(\frac{y_{x x}}{\chi_{\Delta T}^{2}}+v_{x x}\right) \int_{0}^{L}\left(\frac{y_{x} v_{x}}{\chi_{\Delta T}^{2}}+\frac{1}{2} v_{x}^{2}\right) \mathrm{d} x \\
& =\sum_{m=1}^{2} K_{m} \cos \left(\Omega_{m} t+\theta_{m}\right),
\end{aligned}
$$

where the subscript index denotes the partial differential to $x$ and $t$, respectively. $c$ is the viscous damping coefficient; $y A(x)$ is the initial cable profile. Two external distributed loads are denoted by $K_{m} \cos \left(\Omega_{m} t+\theta_{m}\right),(m=1,2)$, where $K_{m}, \Omega_{m}$, and $\theta_{m}$ are excitation amplitudes, frequencies, and phases, respectively. For the sake of simplicity, the influences of temperature on the material properties $E$ and crosssectional area $A$ are neglected.

The following dimensionless parameters and variables are adopted:

$$
\begin{aligned}
v^{*}\left(x^{*}, y^{*}, f^{*}\right) & =\frac{v(x, y, b)}{L}, \\
t^{*} & =\sqrt{\frac{g}{8 b}} t, \\
c^{*} & =\sqrt{\frac{8 b}{g}} \frac{c}{m}, \\
\Theta & =\frac{E A}{H}, \\
K_{m}^{*} & =\frac{K_{m} L}{H}, \\
\Omega_{m}^{*} & =\sqrt{\frac{8 b}{g}} \Omega_{m} .
\end{aligned}
$$

Here, considering the temperature effect, substituting equations (5) into (4) and neglecting asterisk notations, one obtains [20] 


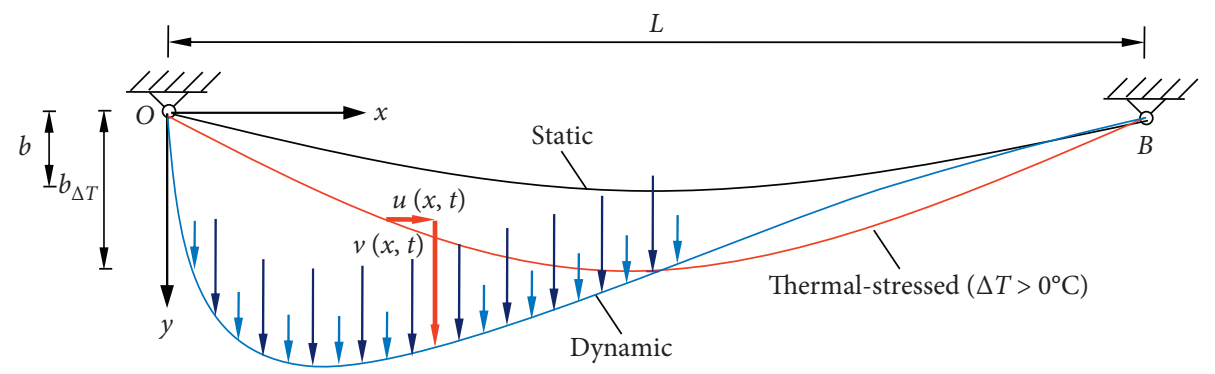

Figure 1: A schematic figure of a suspended cable in thermal environments.

$$
\begin{aligned}
& v_{t t}+c v_{t}-\chi_{\Delta T}^{2} v_{x x}-\Theta\left(\frac{y_{x x}}{\chi_{\Delta T}^{2}}+v_{x x}\right) \int_{0}^{L}\left(\frac{y_{x} v_{x}}{\chi_{\Delta T}^{2}}+\frac{1}{2} v_{x}^{2}\right) \mathrm{d} x \\
& =\sum_{m=1}^{2} K_{m} \cos \left(\Omega_{m} t+\theta_{m}\right) .
\end{aligned}
$$

The in-plane displacement is decomposed by a sum of the generalized coordinates $q_{n}(t)$ and mode shapes $\varphi_{n}(x)$.

$$
v(x, t)=\sum_{n=1}^{\infty} \varphi_{n}(x) q_{n}(t) .
$$

Substituting equation (7) into (6), multiplying the results with $\varphi_{n}(x)$, and integrating the outcomes from 0 to 1 , the following discretized equation is obtained:

$$
\begin{aligned}
& q_{n, t t}+\omega_{n}^{2} q_{n}+2 \mu_{n} q_{n, t}+\sum_{j=1}^{\infty} \sum_{k=1}^{\infty} \Lambda_{j k n} q_{j} q_{k}+\sum_{j=1}^{\infty} \sum_{k=1}^{\infty} \sum_{l=1}^{\infty} \Gamma_{j k l n} q_{j} q_{k} q_{l} \\
& =2 \sum_{m=1}^{2} K_{m n} \cos \left(\Omega_{m} t+\theta_{m}\right),
\end{aligned}
$$

in which coefficients depending on the cable properties with thermal effects are given in Appendix A. An eigenanalysis on the linear parts of equation (8) is performed in Appendix B.

\section{Perturbation Analysis}

The presence of both quadratic and cubic nonlinear terms in equation (8) gives rise to a rich and complex set of responses. A variety of phenomena are associated with the occurrence of the resonance conditions for the system, namely, the primary one, and the order one-half/one-third subharmonic resonances, and the two-order/three-order superharmonic resonances.

Here, considering the quadratic nonlinearity induced by initial curvature, two simultaneous resonances are investigated: the primary and the two-order superharmonic resonances: $2 \Omega_{1} \approx \omega_{1}$ and $\Omega_{2} \approx \omega_{1}$ (Case A), and the two-order superharmonic and the order one-half subharmonic resonances: $2 \Omega_{1} \approx \omega_{1}$ and $\Omega_{2} \approx 2 \omega_{1}$ (Case B). As to a weakly flexible nonlinear system, the method of multiple scales is used to obtain the steady-state solutions of multitype simultaneous resonance responses. Following a similar procedure in [44], the perturbation analysis is presented as follows. follows:

Firstly, the quick and slow times are introduced as

$$
\begin{aligned}
& T_{0}=t, \\
& T_{1}=\varepsilon t, \\
& T_{2}=\varepsilon^{2} t,
\end{aligned}
$$

and the solution of the nonlinear system is in the form of expansion

$$
\begin{aligned}
q_{n}(t ; \varepsilon)= & q_{n 0}\left(T_{0}, T_{1}, T_{2}\right)+\varepsilon q_{n 1}\left(T_{0}, T_{1}, T_{2}\right) \\
& +\varepsilon^{2} q_{n 2}\left(T_{0}, T_{1}, T_{2}\right), \ldots,
\end{aligned}
$$

where $\varepsilon$ is a small nondimensional parameter.

Two detuning parameters $\sigma_{1}$ and $\sigma_{2}$ are introduced for dual harmonic excitations to describe the nearness between them.

Case A:

$$
\begin{aligned}
2 \Omega_{1} & =\omega_{1}+\varepsilon \sigma_{1}, \\
\Omega_{2} & =\omega_{1}+\varepsilon \sigma_{2} .
\end{aligned}
$$

Case B:

$$
\begin{aligned}
2 \Omega_{1} & =\omega_{1}+\varepsilon \sigma_{1}, \\
\Omega_{2} & =2 \omega_{1}+\varepsilon \sigma_{2} .
\end{aligned}
$$

The damping, dual excitations, and quadratic and cubic nonlinearities are rescaled as follows.

Case A:

$$
\begin{aligned}
\mu_{n} & \Rightarrow \varepsilon \mu_{n}, \\
\Lambda_{j k n} & \Rightarrow \varepsilon \Lambda_{j k n}, \\
\Gamma_{j k l n} & \Rightarrow \varepsilon^{2} \Gamma_{j k l n}, \\
K_{1 n} & \Rightarrow \varepsilon^{0} K_{1 n}, \\
K_{2 n} & \Rightarrow \varepsilon^{1} K_{2 n} .
\end{aligned}
$$


Case B:

$$
\begin{aligned}
\mu_{n} & \Rightarrow \varepsilon^{2} \mu_{n}, \\
\Lambda_{j k n} & \Rightarrow \varepsilon \Lambda_{j k n}, \\
\Gamma_{j k l n} & \Rightarrow \varepsilon^{2} \Gamma_{j k l n}, \\
K_{1 n} & \Rightarrow \varepsilon^{1} K_{1 n}, \\
K_{2 n} & \Rightarrow \varepsilon^{1} K_{2 n} .
\end{aligned}
$$

Substituting equations (9)-(10) and (13)-(14) into (8), equating coefficients of the same powers of $\varepsilon$, one obtains the following.

Case A:

$$
\begin{aligned}
& D_{0}^{2} q_{n 0}+\omega_{n}^{2} q_{n 0}=2 K_{1 n} \cos \left(\Omega_{1} T_{0}+\theta_{1}\right) \\
& D_{0}^{2} q_{n 1}+\omega_{n}^{2} q_{n 1}=-2 D_{0} D_{1} q_{n 0}-2 \mu_{n} D_{0} q_{n 0}-\sum_{j=1}^{\infty} \sum_{k=1}^{\infty} \Lambda_{j k n} q_{j 0} q_{k 0}+2 K_{2 n} \cos \left(\Omega_{2} T_{0}+\theta_{2}\right) \\
& D_{0}^{2} q_{n 2}+\omega_{n}^{2} q_{n 2}=-2 D_{0} D_{1} q_{n 1}-\left(2 D_{0} D_{2}+D_{1}^{2}\right) q_{n 0}-\sum_{j=1}^{\infty} \sum_{k=1}^{\infty}\left(\Lambda_{j k n}+\Lambda_{k j n}\right) q_{j 1} q_{k 0}-\sum_{j=1}^{\infty} \sum_{k=1}^{\infty} \sum_{l=1}^{\infty} \Gamma_{j k l n} q_{j 0} q_{k 0} q_{l 0} .
\end{aligned}
$$

Case B:

$$
\begin{aligned}
& D_{0}^{2} q_{n 0}+\omega_{n}^{2} q_{n 0}=0 \\
& D_{0}^{2} q_{n 1}+\omega_{n}^{2} q_{n 1}=-2 D_{0} D_{1} q_{n 0}-\sum_{j=1}^{\infty} \sum_{k=1}^{\infty} \Lambda_{j k n} q_{j 0} q_{k 0}+2 \sum_{m=1}^{2} K_{m n} \cos \left(\Omega_{m} t+\theta_{m}\right) \\
& D_{0}^{2} q_{n 2}+\omega_{n}^{2} q_{n 2}=-2 D_{0} D_{1} q_{n 1}-\left(2 D_{0} D_{2}+D_{1}^{2}+2 \mu_{n} D_{0}\right) q_{n 0}-\sum_{j=1}^{\infty} \sum_{k=1}^{\infty}\left(\Lambda_{j k n}+\Lambda_{k j n}\right) q_{j 1} q_{k 0}-\sum_{j=1}^{\infty} \sum_{k=1}^{\infty} \sum_{l=1}^{\infty} \Gamma_{j k l n} q_{j 0} q_{k 0} q_{l 0} .
\end{aligned}
$$

The generating solution of the first equation in equations (15) and (16) is obtained, respectively.

Case A:

$$
q_{n 0}=A_{n} e^{i \omega_{n} T_{0}}+E_{1 n} e^{i \Omega_{1} T_{0}}+\text { c.c. },
$$

where $E_{1 n}=\left(e^{i \theta_{1}} K_{1 n} /\left(\omega_{n}^{2}-\Omega_{1}^{2}\right)\right)$.

Case B:

$$
q_{n 0}=A_{n} e^{i \omega_{n} T_{0}}+\text { c.c. },
$$

where c.c. denotes the complex conjugate of the preceding terms.

Substituting equations (17) and (18) into the second equation in equations (15) and (16) and eliminating the secular terms from $q_{n 1}$, the solution for $q_{n 1}$ is obtained. Then, secular terms from $q_{n 2}$ in the third equation in equations (15) and (16) could be eliminated by following the same procedure. Finally, the solvability condition is expressed as follows.
Case A:

$$
\begin{aligned}
& -2 i \omega_{n}\left(D_{1} A_{n}+\mu_{n} A_{n}\right)-2 \delta_{n 1} \omega_{1} \bar{F}_{1} e^{i\left(\sigma_{1} T_{1}+2 \theta_{1}\right)} \\
& +\delta_{n 1} K_{21} e^{i\left(\sigma_{2} T_{1}+\theta_{2}\right)}=0
\end{aligned}
$$

Case B:

$$
i\left(D_{2} A_{n}+\mu_{n} A_{n}\right)-4 A_{n} \sum_{j=1}^{\infty} \alpha_{j n} A_{j} \bar{A}_{j}+\delta_{n 1} I_{21} \bar{A}_{1} e^{i\left(\sigma_{2} T_{2}+\theta_{2}\right)}=0
$$

where $\delta_{n 1}$ is the Kronecker delta, and coefficients $\bar{F}_{1}, \alpha_{j n}$, and $I_{21}$ are expressed in Appendix C.

Similarly, the complex amplitude $A_{n}$ can be written in the polar form including the amplitude $a_{n}$ and phase $\beta_{n}$, so equations (19) to (20) will be separated into the real and imaginary parts when $n=1\left(a_{n}\right.$ decay for $\left.n \geq 2\right)$.

Case A: 


$$
\begin{array}{r}
\omega_{1} a_{1}^{\prime}+\omega_{1} \mu_{1} a_{1}+2 \omega_{1} \bar{F}_{1} \sin \left(\sigma_{1} T_{1}+2 \theta_{1}-\beta_{1}\right)-K_{21} \sin \left(\sigma_{2} T_{1}+\theta_{2}-\beta_{1}\right)=0 \\
\omega_{1} a_{1} \beta_{1}^{\prime}-2 \omega_{1} \bar{F}_{1} \cos \left(\sigma_{1} T_{1}+2 \theta_{1}-\beta_{1}\right)+K_{21} \cos \left(\sigma_{2} T_{1}+\theta_{2}-\beta_{1}\right)=0 .
\end{array}
$$

Case B:

$$
\begin{aligned}
a_{1}^{\prime}+\mu_{1} a_{1}+I_{21} a_{1} \sin \left(\sigma_{2} T_{2}+\theta_{2}-2 \beta_{1}\right) & =0, \\
a_{1}\left(\beta_{1}^{\prime}+\alpha_{11} a_{1}^{2}\right)+I_{21} a_{1} \cos \left(\sigma_{2} T_{2}+\theta_{2}-2 \beta_{1}\right) & =0 .
\end{aligned}
$$

In Case A, it leads to the requirement that $\sigma_{2}=\sigma_{1}$ and $\Omega_{2}=2 \Omega_{1}$ as to the steady-state response, and one defines $\sigma=\sigma_{1}=\sigma_{2}$ and $\gamma=\sigma T_{1}-\beta_{1}$. Accordingly, the governing equations are obtained.

Case A:

$$
\begin{aligned}
& \omega_{1} \mu_{1} a_{1}+2 \omega_{1} \bar{F}_{1} \sin \left(\gamma+2 \theta_{1}\right)-K_{21} \sin \left(\gamma+\theta_{2}\right)=0, \\
& \omega_{1} a_{1} \sigma-2 \omega_{1} \bar{F}_{1} \cos \left(\gamma+2 \theta_{1}\right)+K_{21} \cos \left(\gamma+\theta_{2}\right)=0 .
\end{aligned}
$$

Similarly, in Case B, one defines $\sigma=\left(\sigma_{2} / 2\right)$ and $\gamma=$ $\sigma T_{2}-\beta_{1}$ and obtains the following.

Case B:

$$
\begin{array}{r}
\mu_{1} a_{1}+I_{21} a_{1} \sin \left(2 \gamma+\theta_{2}\right)=0, \\
a_{1}\left(\sigma+\alpha_{11} a_{1}^{2}\right)+I_{21} a_{1} \cos \left(2 \gamma+\theta_{2}\right)=0 .
\end{array}
$$

Hence, the relationship between the detuning parameter $\sigma$ and the response amplitude $a_{1}$ could be expressed as follows.

Case A:

$$
a_{1}= \pm \frac{1}{\omega_{1}} \sqrt{\sigma^{2}+\mu_{1}^{2}}\left(2 \omega_{1} \bar{F}_{1}-K_{21}\right)
$$

Substituting $\bar{F}_{1}$ into equation (25), one obtains

$$
a_{1}= \pm \frac{16 \Lambda_{111} K_{11}^{2}-9 \omega_{1}^{4} K_{21}}{9 \omega_{1}^{5} \sqrt{\sigma^{2}+\mu_{1}^{2}}}
$$

where $\theta_{2}=2 \theta_{1}$.

Case B:

$$
a_{1}^{2}=\frac{-\sigma \pm \sqrt{I_{21}^{2}-\mu_{1}^{2}}}{\alpha_{11}} .
$$

Introducing two small disturbances $\left(a_{1}=a_{10}+a_{11}\right.$ and $\left.\gamma=\gamma_{0}+\gamma_{1}\right)$, substituting them into (21) and (22), expanding for small $a_{11}$ and $\gamma_{1}$, noticing that $a_{10}$ and $\gamma_{0}$ satisfy equations (23) and (24), and finally keeping linear terms in $a_{11}$ and $\gamma_{1}$, one obtains the following.

Case A:

$$
\begin{aligned}
& a_{11}^{\prime}=-\mu_{1} a_{11}+\left[-2 \bar{F}_{1} \cos \left(\gamma_{0}+2 \theta_{1}\right)+\frac{K_{21}}{\omega_{1}} \cos \left(\gamma_{0}+\theta_{2}\right)\right] \gamma_{1}, \\
& \gamma_{1}^{\prime}=\left[\frac{2 \bar{F}_{1}}{a_{10}} \sin \left(\gamma_{0}+2 \theta_{1}\right)-\frac{K_{21}}{a_{10} \omega_{1}} \sin \left(\gamma_{0}+\theta_{2}\right)\right] \gamma_{1} .
\end{aligned}
$$

Case B:

$$
\begin{aligned}
a_{11}^{\prime}= & {\left[-\mu_{1}-I_{21} \sin \left(2 \gamma_{0}+\theta_{2}\right)\right] a_{11} } \\
& +\left[-2 I_{21} a_{10} \cos \left(2 \gamma_{0}+\theta_{2}\right)\right] \gamma_{1}, \\
\gamma_{1}^{\prime}= & 2 \alpha_{11} a_{10} a_{11}-2 I_{21} \sin \left(2 \gamma_{0}+\theta_{2}\right) \gamma_{1},
\end{aligned}
$$

where the stability of the nontrivial solutions is determined by investigating the eigenvalues of the coefficients matrix.

\section{Numerical Examples and Discussions}

The following physical parameters are adopted in the numerical examples, as shown in Table 1. Based on the parabolic assumption of the suspended cables' configuration, four sag-to-span ratios (e.g., 0.0025, 0.006, 0.01, and 0.015) are adopted to exhibit the influence of temperature on the vibration behaviors. It was shown that the nonlinear dynamic behaviors of the suspended cable are not sensitive to the temperature-dependent materials between $\left[-40^{\circ} \mathrm{C}, 40^{\circ} \mathrm{C}\right]$, such as Young's modulus [23]. Here, material properties are assumed to be independent of temperature variations, and the cross-sectional area remains plane and uniform in thermal environments.

As illustrated in the previous sections, the infinite-dimensional model is considered. Nevertheless, in the following numerical examples, just a single-degree-of-freedom model is adopted for simplicity. Table 2 gives the tension variation factor, natural frequency, and quadratic and cubic nonlinear coefficients in the warming $\left(\Delta T=40^{\circ} \mathrm{C}\right)$ and cooling $\left(\Delta T=-40^{\circ} \mathrm{C}\right)$ conditions. The temperature effects on linear (natural frequency) and nonlinear (quadratic and cubic) coefficients are closely related to the sag-to-span ratios. To be more specific, both positive and negative relationships between temperature changes and natural frequencies are found. If the sag-to-span ratio is tiny, for example, 0.0025 and 0.006 , the temperature effects on the cubic coefficients seem to be neglected. As an increase in the sag-to-span ratio, both quadratic and cubic nonlinearity coefficients are increased (decreased) in the warming (cooling) condition. Due to the variations of these parameters and coefficients, the influences of temperature on vibration behaviors are discussed.

The response amplitudes $a_{1}$ as functions of two excitation amplitudes $K_{11}$ and $K_{21}$ for this nonlinear system at a given detuning parameter $\sigma$ are presented in Figures 2 and 3 by solving equation (26), respectively. First of all, it is assumed that the excitation amplitude $K_{21}$ is a constant 
TABLE 1: Notations and values of physical parameters.

\begin{tabular}{lc}
\hline Physical meaning (notation) & Value (unit) \\
\hline Density $(\rho)$ & $7800\left(\mathrm{~kg} / \mathrm{m}^{3}\right)$ \\
Cable span $(L)$ & $200(\mathrm{~m})$ \\
Young's modulus $(E)$ & $2.0 \times 10^{11}(\mathrm{~Pa})$ \\
Area of cross section $(A)$ & $7.069 \times 10^{-2}\left(\mathrm{~m}^{2}\right)$ \\
Thermal expansion coefficient $(\alpha)$ & $1.2 \times 10^{-5}\left(/{ }^{\circ} \mathrm{C}\right)$ \\
Temperature variations $(\Delta T)$ & $-40,0,40\left({ }^{\circ} \mathrm{C}\right)$ \\
Damping ratio $(\mu)$ & $0.005(-)$ \\
Sag-to-span ratio $(f)$ & $0.0025,0.006,0.01,0.015(-)$ \\
\hline
\end{tabular}

TABLE 2: Values of corresponding parameters for the first symmetric mode.

\begin{tabular}{|c|c|c|c|c|c|}
\hline$f$ & $\Delta T\left({ }^{\circ} \mathrm{C}\right)$ & $\chi_{\Delta T}^{2}$ & $\omega_{n}^{2}$ & $\Lambda_{n n n}$ & $\Gamma_{n n n n}$ \\
\hline \multirow{3}{*}{0.0025} & -40 & 1.1345 & 11.2803 & 71.86 & 13771.7 \\
\hline & 0 & 1.0000 & 9.9769 & 81.52 & 13771.8 \\
\hline & +40 & 0.8661 & 8.6912 & 94.12 & 13771.8 \\
\hline \multirow{3}{*}{0.006} & -40 & 1.2783 & 13.3356 & 314.66 & 30607.1 \\
\hline & 0 & 1.0000 & 11.0444 & 401.86 & 30618.3 \\
\hline & +40 & 0.7464 & 9.4694 & 537.45 & 30690.5 \\
\hline \multirow{3}{*}{0.01} & -40 & 1.3713 & 16.4205 & 812.71 & 51085.7 \\
\hline & 0 & 1.0000 & 15.2646 & 1112.82 & 51571.5 \\
\hline & +40 & 0.7352 & 17.0703 & 1538.31 & 55321.5 \\
\hline \multirow{3}{*}{0.015} & -40 & 1.3407 & 22.9867 & 1826.90 & 77269.8 \\
\hline & 0 & 1.0000 & 27.0093 & 2539.48 & 87587.5 \\
\hline & +40 & 0.7930 & 33.6275 & 3980.64 & 158065.0 \\
\hline
\end{tabular}

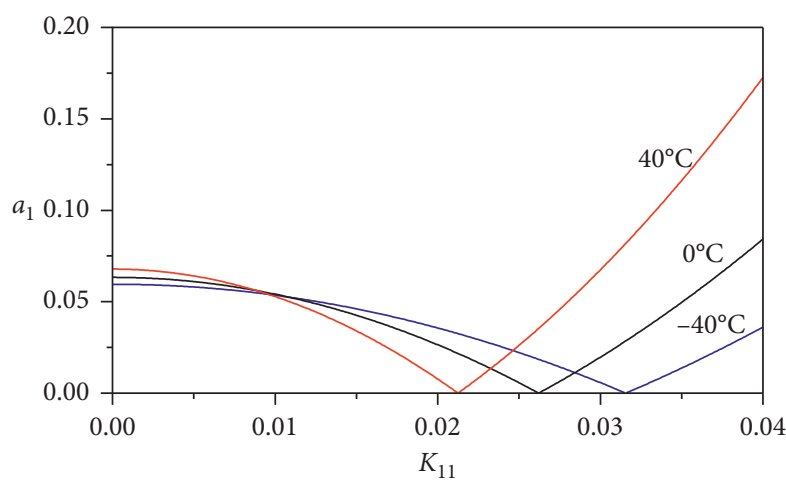

(a)

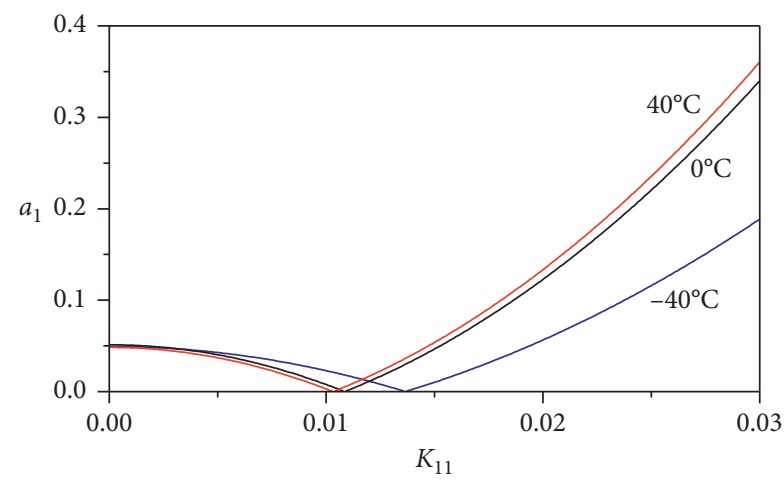

(c)

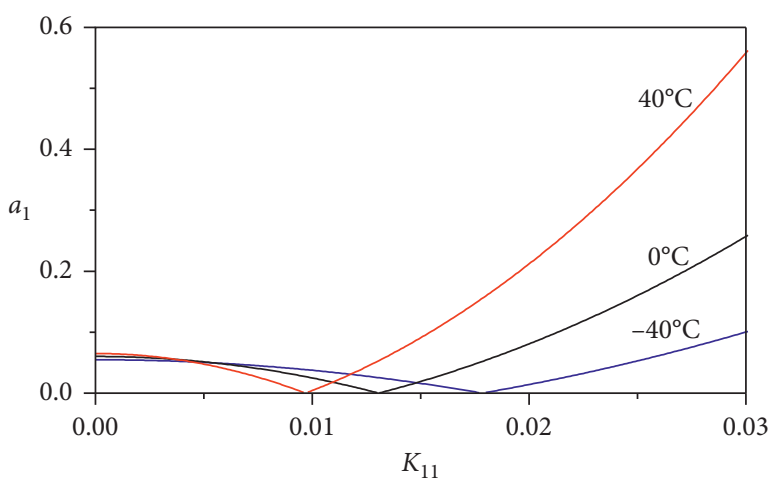

(b)

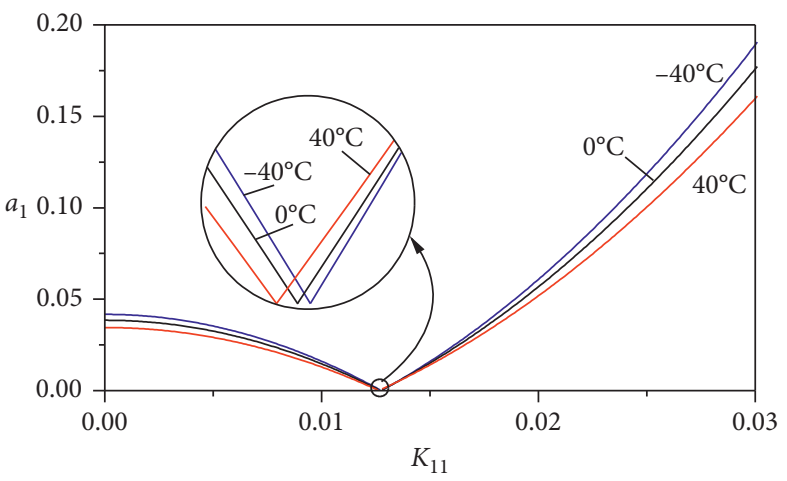

(d)

FIgURE 2: Temperature effects on relationships between excitation amplitude $K_{11}$ and response amplitude $a_{1}$ when $K_{21}=0.0001$ in Case (A). (a) $f=0.0025$, (b) $f=0.006$, (c) $f=0.010$, and (d) $f=0.015$. 


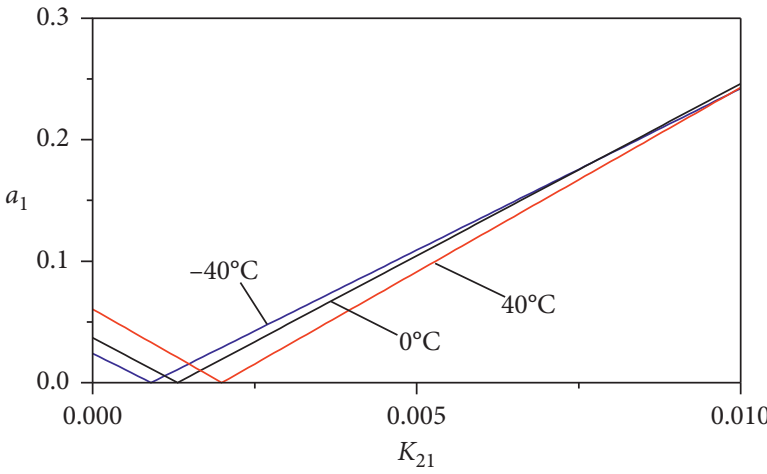

(a)

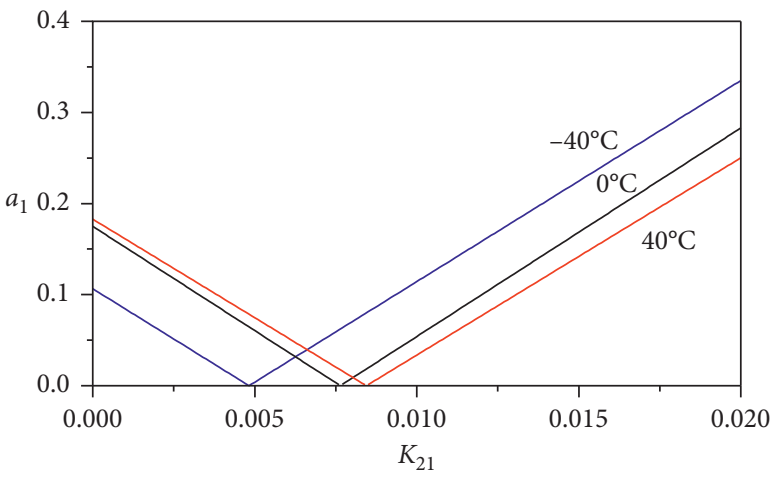

(c)

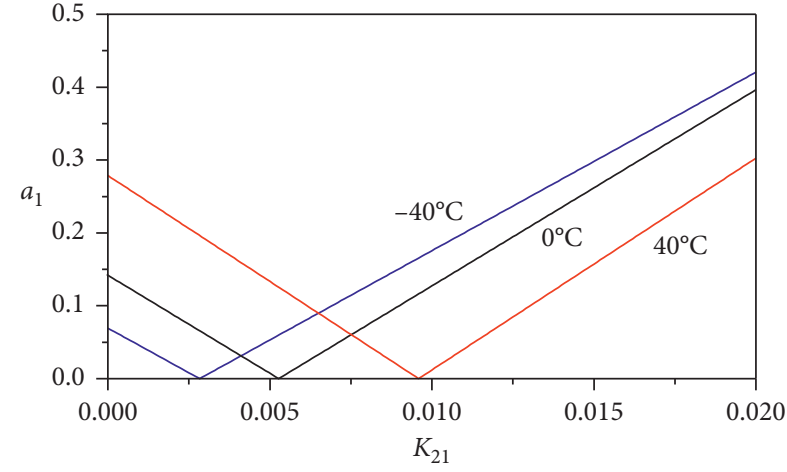

(b)

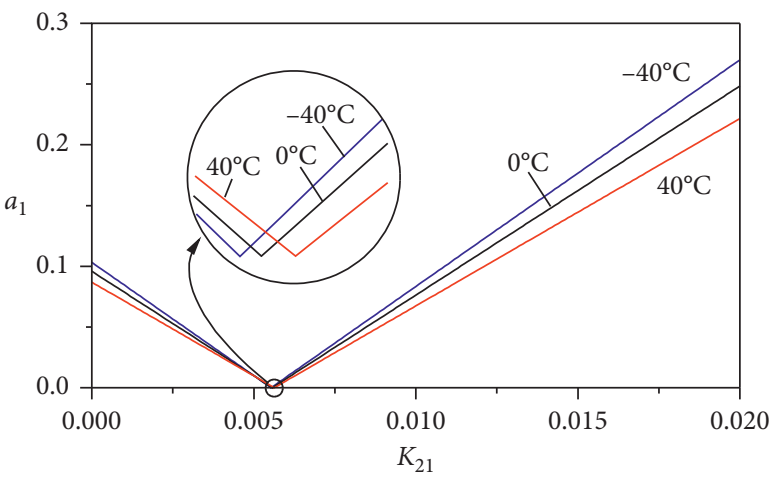

(d)

Figure 3: Temperature effects on relationships between excitation amplitude $K_{21}$ and response amplitude $a_{1}$ when $K_{11}=0.005$ in Case (A). (a) $f=0.0025$, (b) $f=0.006$, (c) $f=0.010$, and (d) $f=0.015$.

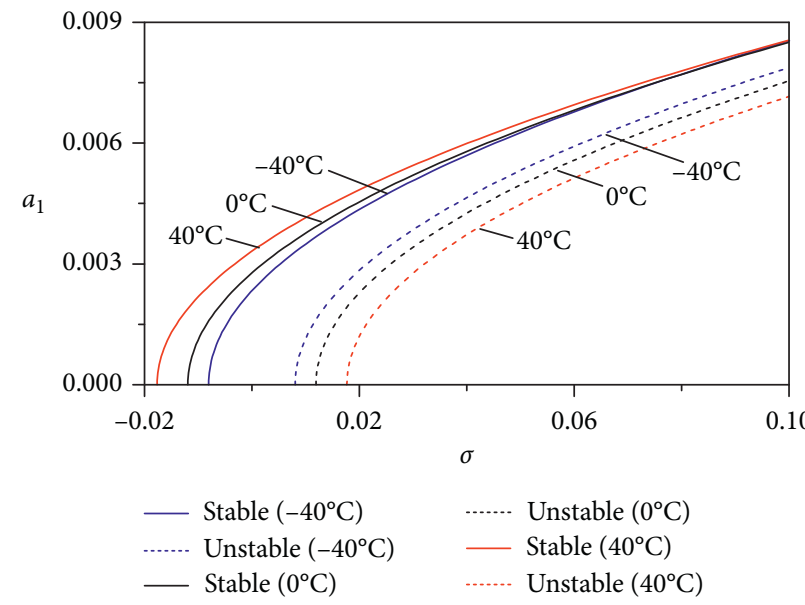

(a)

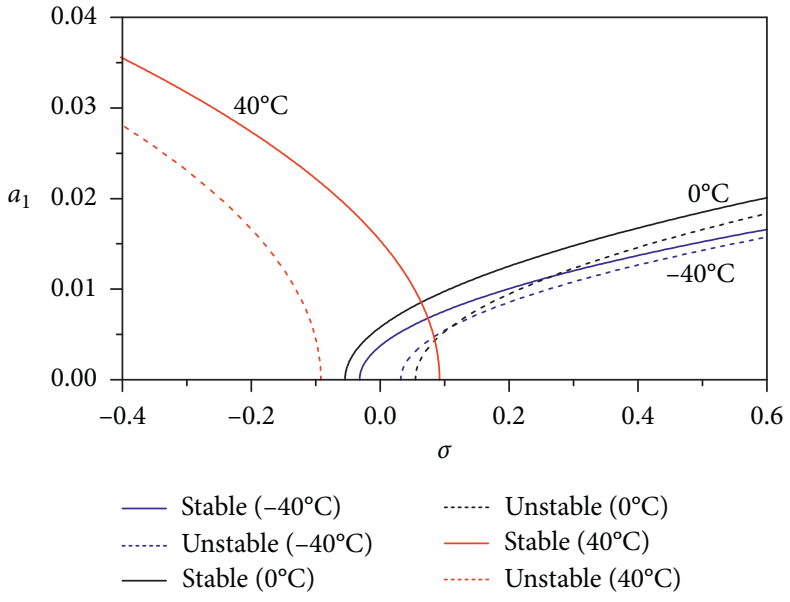

(b)

Figure 4: Continued. 


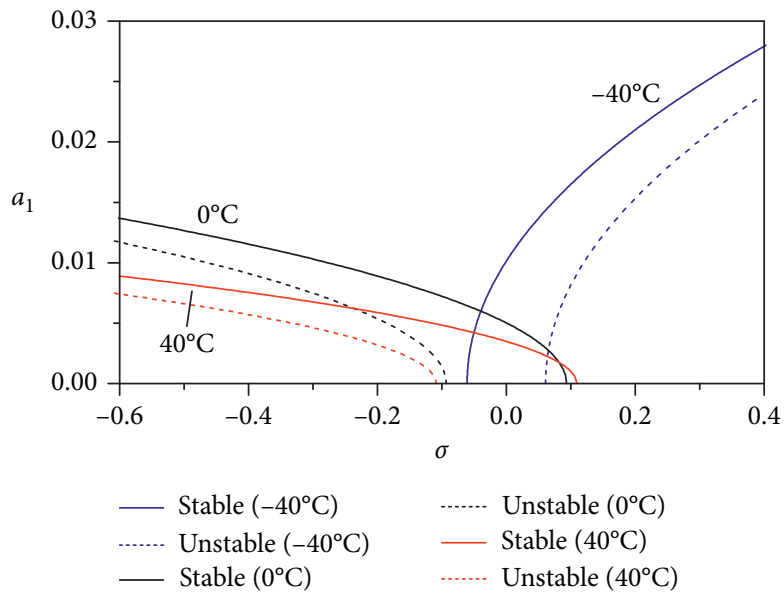

(c)

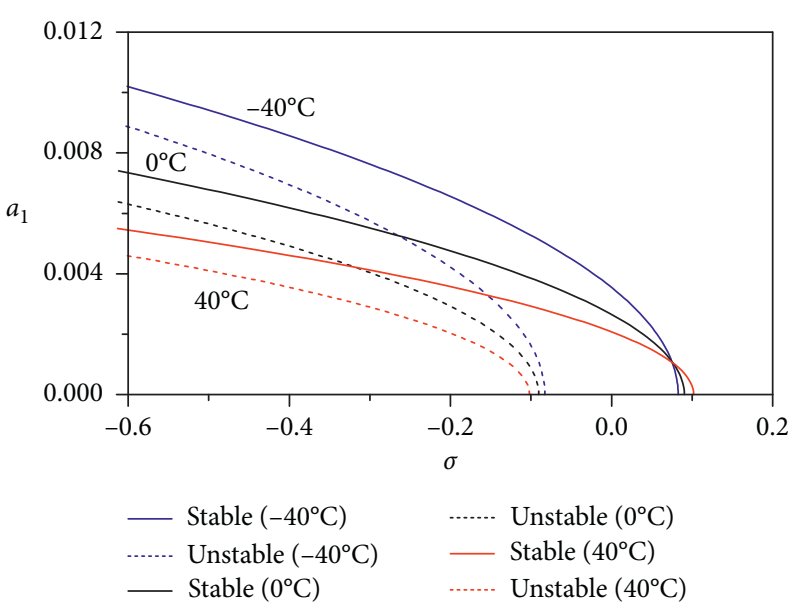

(d)

FIgURE 4: Temperature effects on relationships between detuning parameter $\sigma$ and response amplitude $a$ when $K_{21}=K_{11}=0.015$ in Case (B). (a) $f=0.0025$, (b) $f=0.006$, (c) $f=0.010$, and (d) $f=0.015$.
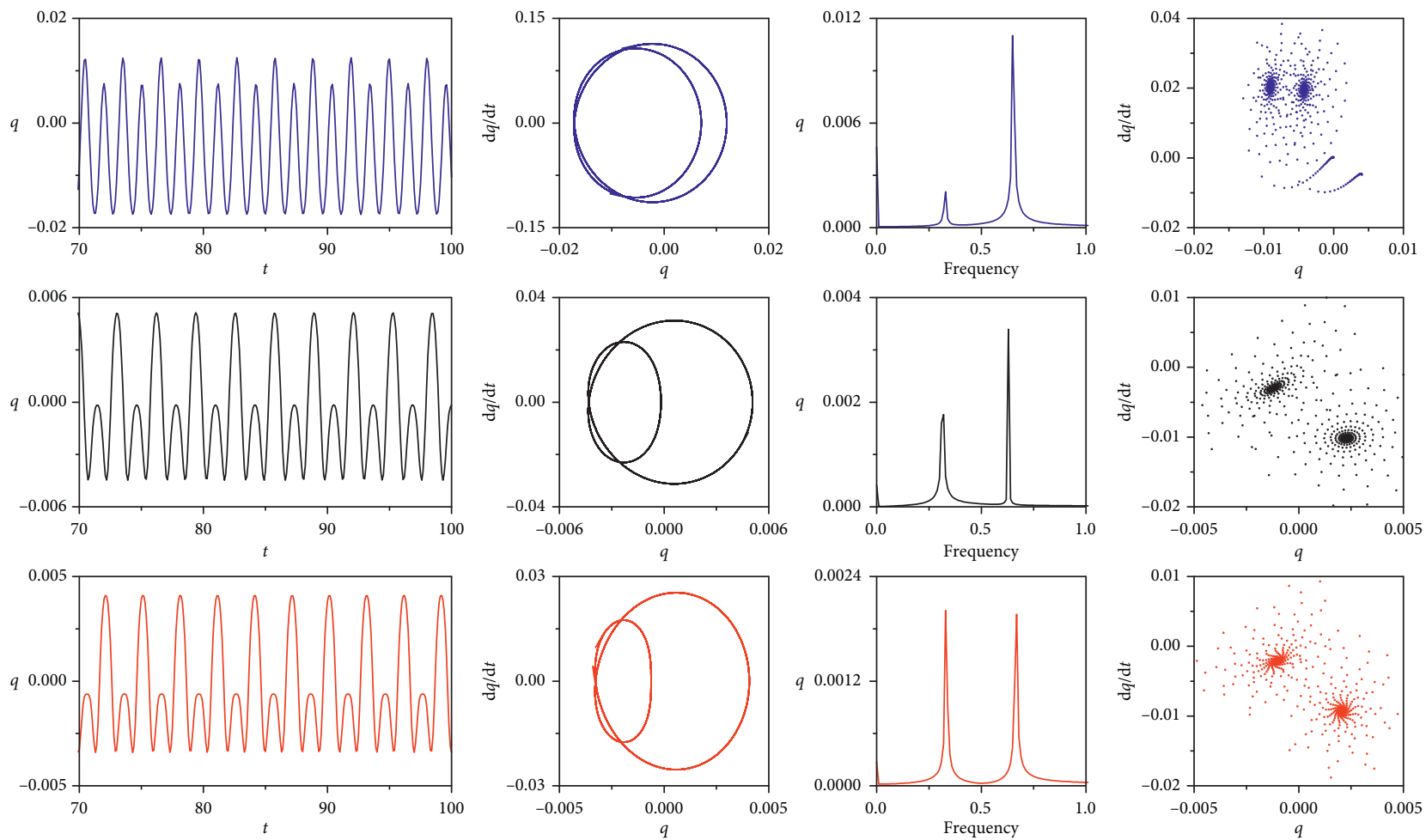

Figure 5: Temperature effects on time history curves, phase portraits, frequency spectrum, and Poincaré sections when $f=0.01, \sigma=0.05$, and $K_{21}=K_{11}=0.015$ in Case (B). (a) $40^{\circ} \mathrm{C}$, (b) $0^{\circ} \mathrm{C}$, and (c) $-40^{\circ} \mathrm{C}$.

(0.0001), and the detuning parameter $\sigma$ is zero. As shown in Figure 2, with an increase in $K_{11}$ from zero, the response amplitude $a_{1}$ decreases to zero at the beginning and then increases after the inflection point.

To be more specific, Figure 2(a) shows that the response amplitude $a_{1}$ increases (decreases) in the warming (cooling) condition when $K_{11}<0.01$. On the contrary, provided that
$0.01<K_{11}<0.02$, the response amplitude $a_{1}$ is reduced in the warming condition. The inflection point will be exhibited earlier in the warming condition when the excitation amplitude increases. Equation (26) shows that the inflection point is exhibited when $K_{11}=\sqrt{\left(9 \omega_{1}^{4} K_{21} / 16 \Lambda_{111}\right)}$. Here, as to the cable with $f=0.0025$, the natural frequency increases 
when $\Delta T=-40^{\circ} \mathrm{C}$ and decreases when $\Delta T=40^{\circ} \mathrm{C}$. The quadratic coefficient will increase significantly when the temperature is increased. Then, if $K_{11}$ keeps increasing, the amplitude of responses $a_{1}$ increases accordingly in the warming condition.

As shown in Figure 2(b), there are some similarities of the temperature effects on the vibration behaviors between $f=0.0025$ and 0.006 . Because the increase of quadratic coefficient is much more significant than the increase of natural frequency, it is noted that all the inflection points are exhibited in a smaller value of $K_{11}$. However, if $f$ is chosen as 0.01 , some differences are exhibited. The influences of temperature on the vibration characteristics seem more obvious in the cooling condition than in the warming one. The primary reason comes from the natural frequency with thermal effects, because it increases in both warming and cooling conditions. Furthermore, in the case of $f=0.015$, both the natural frequency and the quadratic coefficient increase (decrease) with the increase (decrease) of temperature. Therefore, no matter before or after the inflection point, the response amplitudes $a_{1}$ will be reduced in the warming condition. Here, it should be pointed out that the temperature effects on the vibration behaviors seem reduced significantly when $f$ is chosen as 0.015 .

Moreover, suppose that the first excitation amplitude $K_{11}$ is a constant. In that case, the temperature effects on the relationships between the excitation amplitude $K_{21}$ and the response one $a_{1}$ are described in Figure 3 for a given detuning parameter. As the previous case, the response amplitude $a_{1}$ decreases initially, followed by an increase with $K_{21}$. Similarly, there is an inflection point in the response amplitudes, and it is exhibited when $K_{21}=\left(16 \Lambda_{111} K_{11}^{2} / 9 \omega_{1}^{4}\right)$. On the contrary, the point seems to be delayed in the warming condition when $K_{21}$ increases from zero.

As to the case of $f=0.0025,0.006$, and 0.01 , the response amplitude $a_{1}$ increases in the warming condition before the inflection point. Nevertheless, after the inflection point, $a_{1}$ decreases in the warming condition. It is also observed that the discrepancies between different conditions are reduced when $K_{21}$ keeps increasing in the latter. As to the case of $f=0.015$, no significant change on the inflection point could be observed. The response amplitude $a_{1}$ would be reduced in the warming condition no matter before or after the inflection point.

Furthermore, the temperature effects on relationships between detuning parameter $\sigma$ and response amplitude $a$ are described in Figure 4 as to Case B. Similarly, four cases are discussed, and two excitation amplitudes are constant: $K_{21}=K_{11}=0.015$. Solid lines denote the stable steady-state solutions, and dashed lines denote the unstable ones. Excited by certain harmonic excitations, the suspended cable exhibits softening or hardening behaviors depending on the contribution of quadratic and cubic nonlinearities and the corresponding configurations.

In the case of $f=0.0025$ in Figure 4(a), all branches are bent to right, and the nonlinear system presents a hardening-type nonlinearity. Provided that the excitation frequency continues to decrease from -0.1 , the trivial fixed point loses its stability at the Pitchfork bifurcation. In the cooling condition, the bifurcation point seems to be delayed, and the resonant range between two Pitchfork bifurcations is reduced significantly $\left(\Delta T=-40^{\circ} \mathrm{C}\right)$. With an increase in the detuning parameter $\sigma$, the influences of temperature on the stable solutions seem reduced more and more.

Regarding the dynamical behaviors when $f=0.006$ in Figure 4(b), the resonant curves show that the nonlinear system exhibits a hardening-type behavior when no temperature is considered. The lower the temperature drops (e.g., $\Delta T=-40^{\circ} \mathrm{C}$ ), the more the curves are bending to the right, and the response amplitudes are reduced. However, if the temperature increases to $40^{\circ} \mathrm{C}$, a softening-type characteristic is exhibited due to the temperature effects on the quadratic nonlinearity coefficient.

However, when $f$ is chosen as 0.01 in Figure 4(c), at a specific low temperature (e.g., $\Delta T=-40^{\circ} \mathrm{C}$ ), the nonlinear system exhibits a softening-type behavior. In contrast, for sufficiently high temperature (e.g., $\Delta T=40^{\circ} \mathrm{C}$ ), the nonlinear behavior is changed to a softening type. As shown in Figure 4(d) ( $f=0.015)$, all branches are bent to the left, and they all present a softening-type nonlinearity in diffident thermal environments. It is observed that the nonlinear system predicts a weaker softening behavior in the cooling condition.

Moreover, details of the nonlinear system responses are described in Figure 5 through some time traces, phase-plane portraits, fast Fourier transforms (FFTs), and Poincaré sections in the case of $f=0.01, \sigma=0.05$, and $K_{21}=K_{11}=0.015$. On the one hand, temperature increasing causes a smaller peak of the response amplitude. On the other hand, as shown in the phase portraits, two circles could be observed in different conditions. To verify the frequency components of the time traces, the fast Fourier transform (FFT) is adopted here. A FFT analysis suggests that the motion mainly comprises two frequencies. At the same time, the Periodic- 2 motions are also confirmed, because two isolated dots in the Poincaré sections could be observed. Nevertheless, the peak value of the response amplitude is sensitive to temperature variations, and the periodic motions seem to be independent of thermal effects.

\section{Conclusions}

The sensitivities of linear (natural frequency) and nonlinear (quadratic and cubic) coefficients to temperature variations are different, and they are closely related to the suspended cable's sag-to-span ratios. In general, as to a string with a very large initial tension force and a suspended cable with a very large sag, the temperature's influence on their vibration behaviors seems to be negligible. The temperature may increase or decrease the response amplitudes, depending on the excitation amplitude and the initial tension force. Considering two excitation amplitudes, if one keeps constant, the response amplitude decreases to zero firstly and then increases after the inflection point when another excitation amplitude increases continuously. The inflection point is shifted due to thermal effects and exhibited at a smaller or larger excitation amplitude in thermal environments. Temperature variations may affect the hardening or 
softening spring characteristics. Even an opposite behavior may be exhibited in warming/cooling conditions due to the temperature effects on the cable tension force. Temperature variations significantly affect the response amplitudes and affect the stable region of the nonlinear system. The resonant range between two Pitchfork bifurcations seems reduced when the temperature is decreasing. It is found out that the response amplitude is very sensitive to thermal effects, but the periodic motions seem to be independent of temperature in these cases.

\section{Appendix}

\section{A. The Coefficients Depending on the Cable Properties with Thermal Effect in Equation (8)}

$$
\begin{aligned}
\mu_{n} & =\frac{1}{2} c \\
K_{m n} & =\frac{K_{m}}{2} \int_{0}^{1} \varphi_{n} \mathrm{~d} x, \\
\Lambda_{j k l n} & =-\frac{\Theta}{2} \int_{0}^{1}\left[\varphi_{k, x x} \int_{0}^{1} \varphi_{j, x} \varphi_{l, x} \mathrm{~d} x\right] \varphi_{n} \mathrm{~d} x, \\
\omega_{n}^{2} & =-\chi_{\Delta T}^{2} \int_{0}^{1} \varphi_{n, x x} \varphi_{n} \mathrm{~d} x-\frac{\Theta}{\chi_{\Delta T}^{4}} \int_{0}^{1} y_{x x}\left[\int_{0}^{1} y_{x} \varphi_{n, x} \mathrm{~d} x\right] \varphi_{n} \mathrm{~d} x,
\end{aligned}
$$$$
\Gamma_{j k n}=-\frac{\Theta}{\chi_{\Delta T}^{2}} \int_{0}^{1}\left[\varphi_{k, x x} \int_{0}^{1} y_{x} \varphi_{j, x} \mathrm{~d} x\right] \varphi_{n} \mathrm{~d} x-\frac{\Theta}{2 \chi_{\Delta T}^{2}} \int_{0}^{1}\left[y_{x x} \int_{0}^{1} \varphi_{k, x} \varphi_{j, x} \mathrm{~d} x\right] \varphi_{n} \mathrm{~d} x .
$$

\section{B. An Eigenvalue Analysis on the Linear Parts of Equation (8)}

By solving the transcendental equations, one obtains the inplane symmetric mode frequencies

$$
\tan \left(\frac{\omega_{n}}{2 \chi_{\Delta T}}\right)=\frac{\omega_{n}}{2 \chi_{\Delta T}}-\frac{4}{\lambda_{\Delta T}^{2}}\left(\frac{\omega_{n}}{2 \chi_{\Delta T}}\right)^{3}, \quad(n=1,3,5, \ldots),
$$

where

$$
\begin{aligned}
\lambda_{\Delta T}^{2} & =\frac{\lambda^{2}}{\chi_{\Delta T}^{6},} \\
\text { and } \quad \lambda^{2} & =\left(\frac{m g L}{H}\right)^{2} \frac{E A}{H},
\end{aligned}
$$

and the in-plane symmetric mode shapes are expressed as

$$
\begin{aligned}
\varphi_{n}(x)= & \xi_{n}\left[1-\cos \left(\frac{\omega_{n}}{\chi_{\Delta T}} x\right)\right. \\
& \left.-\tan \left(\frac{\omega_{n}}{2 \chi_{\Delta T}}\right) \sin \left(\frac{\omega_{n}}{\chi_{\Delta T}} x\right)\right], \quad(n=1,3,5, \ldots),
\end{aligned}
$$

where $\xi_{n}$ would be ascertained through $\int_{0}^{1} \varphi_{n}^{2} \mathrm{~d} x=1$.

In-plane antisymmetric mode shapes and frequencies are expressed as follows, respectively:

$$
\begin{aligned}
\omega_{n} & =\chi_{\Delta T} n \pi, \\
\varphi_{n}(x) & =\sqrt{2} \sin (n \pi x), \quad n=2,4,6, \ldots,
\end{aligned}
$$

where all these mode shapes are independent of temperature effects. 


\section{The Coefficients in Equations (19)-(20)}

$$
\begin{aligned}
\bar{F}_{m} & =\frac{1}{2 \omega_{m}} \sum_{j=1}^{\infty} \sum_{k=1}^{\infty} \Lambda_{j k m} \bar{E}_{1 j} \bar{E}_{1 k}, \\
\bar{E}_{m n} & =\frac{K_{m n}}{\omega_{n}^{2}-\Omega_{m}^{2}}, \\
I_{m l} & =\frac{1}{2 \omega_{l}} \sum_{j=1}^{\infty} \bar{\Lambda}_{j l l} \bar{E}_{m j}, \\
\alpha_{j n} & =\frac{1}{8\left(1+\delta_{j n}\right) \omega_{n}}\left\{-\bar{\Gamma}_{j j n n}+\sum_{k=1}^{\infty}\left[\bar{\Lambda}_{k n n} \bar{\Lambda}_{j j k} S_{k n n}+\bar{\Lambda}_{j k n} \bar{\Lambda}_{j n k}\left(R_{k j n}+S_{k j n}\right)\right]\right\}, \\
\bar{\Gamma}_{j k l n} & =\Gamma_{j k l n}+\Gamma_{k j l n}+\Gamma_{j l k n}+\Gamma_{k l j n}+\Gamma_{l j k n}+\Gamma_{l k j n}, \bar{\Lambda}_{j k n}=\Lambda_{j k n}+\Lambda_{k j n}, \\
R_{n j k} & =\frac{1}{\omega_{n}^{2}-\left(\omega_{j}+\omega_{k}\right)^{2}}, \\
S_{n j k} & =\frac{1}{\omega_{n}^{2}-\left(\omega_{j}-\omega_{k}\right)^{2}} .
\end{aligned}
$$

\section{Data Availability}

No data were used to support the findings of this study.

\section{Conflicts of Interest}

The authors declare that they have no conflicts of interest.

\section{Acknowledgments}

This research was funded by the National Natural Science Foundation of China (NSFC) (no. 11602089) and the Promotion Program for Young and Middle-aged Teacher in Science and Technology Research of Huaqiao University (no. ZQN-YX505).

\section{References}

[1] V. Settimi, E. Saetta, and G. Rega, "Nonlinear dynamics of a third-order reduced model of thermomechanically coupled plate under different thermal excitations," Meccanica, vol. 55, no. 12, pp. 2451-2473, 2020.

[2] Y. Xia, B. Chen, S. Weng, Y.-Q. Ni, and Y.-L. Xu, "Temperature effect on vibration properties of civil structures: a literature review and case studies," Journal of Civil Structural Health Monitoring, vol. 2, no. 1, pp. 29-46, 2012.

[3] Y.-W. Zhang, S. Hou, Z. Zhang et al., "Nonlinear vibration absorption of laminated composite beams in complex environment," Nonlinear Dynamics, vol. 99, no. 4, pp. 2605-2622, 2020.

[4] A. Zippo, M. Barbieri, G. Iarriccio, and F. Pellicano, "Nonlinear vibrations of circular cylindrical shells with thermal effects: an experimental study," Nonlinear Dynamics, vol. 99, no. 1, pp. 373-391, 2020.
[5] A. M. Dehrouyeh-Semnani, E. Dehdashti, M. R. H. Yazdi, and M. Nikkhah-Bahrami, "Nonlinear thermo-resonant behavior of fluid-conveying FG pipes," International Journal of Engineering Science, vol. 144, Article ID 103141, 2019.

[6] Z. Shen, H. Li, X. Liu, and G. Hu, "Thermal-structural dynamic analysis of a satellite antenna with the cable-network and hoop-truss supports," Journal of Thermal Stresses, vol. 42, no. 11, pp. 1339-1356, 2019.

[7] Y. Song, Z. Liu, A. Rønnquist, P. Navik, and Z. Liu, "Contact wire irregularity stochastics and effect on high-speed railway pantograph-catenary interactions," IEEE Transactions on Instrumentation and Measurement, vol. 69, no. 10, pp. 8196-8206, 2020.

[8] Y. Song, Z. Wang, Z. Liu, and R. Wang, "A spatial coupling model to study dynamic performance of pantograph-catenary with vehicle-track excitation," Mechanical Systems and Signal Processing, vol. 151, Article ID 107336, 2021.

[9] Y. Song, Z. Liu, H. Ouyang, H. Wang, and X. Lu, "Sliding mode control with PD sliding surface for high-speed railway pantograph-catenary contact force under strong stochastic wind field," Shock and Vibration, vol. 2017, Article ID 4895321, 16 pages, 2017.

[10] Y. Bian and Z. Gao, "Nonlinear vibration control for flexible manipulator using 1:1 internal resonance absorber," Journal of Low Frequency Noise, Vibration and Active Control, vol. 37, no. 4, pp. 1053-1066, 2018.

[11] G. Rega, "Nonlinear vibrations of suspended cables-Part I: modeling and analysis," Applied Mechanics Reviews, vol. 57, no. 6, pp. 443-478, 2004.

[12] G. Rega, "Nonlinear vibrations of suspended cables-Part II: deterministic phenomena," Applied Mechanics Reviews, vol. 57, no. 6, pp. 479-514, 2004.

[13] N. Bouaanani and P. Marcuzzi, "Finite difference thermoelastic analysis of suspended cables including extensibility and 
large sag effects," Journal of Thermal Stresses, vol. 34, no. 1, pp. 18-50, 2011.

[14] S. Montassar, O. B. Mekki, and G. Vairo, "On the effects of uniform temperature variations on stay cables," Journal of Civil Structural Health Monitoring, vol. 5, no. 5, pp. 735-742, 2015.

[15] M. Rezaiee-Pajand, M. Mokhtari, and A. R. Masoodi, “A novel cable element for nonlinear thermo-elastic analysis," Engineering Structures, vol. 167, pp. 431-444, 2018.

[16] F. Treyssède, "Finite element modeling of temperature load effects on the vibration of local modes in multi-cable structures," Journal of Sound and Vibration, vol. 413, pp. 191-204, 2018.

[17] W. M. Zhang, G. M. Tian, and Z. Liu, "Analytical Study of uniform thermal effects on cable configuration of a suspension bridge during construction," Journal of Bridge Engineering, vol. 24, no. 11, Article ID 4019104, 2019.

[18] H. M. Irvine, Cable Structures, The MIT Press, Cambridge, MA, USA, 1981.

[19] F. Treyssède, "Free linear vibrations of cables under thermal stress," Journal of Sound and Vibration, vol. 327, no. 1-2, pp. 1-8, 2009.

[20] M. Lepidi and V. Gattulli, "Static and dynamic response of elastic suspended cables with thermal effects," International Journal of Solids and Structures, vol. 49, no. 9, pp. 1103-1116, 2012.

[21] Y. Zhao, J. Peng, Y. Zhao, and L. Chen, "Effects of temperature variations on nonlinear planar free and forced oscillations at primary resonances of suspended cables," Nonlinear Dynamics, vol. 89, no. 4, pp. 2815-2827, 2017.

[22] Y. Zhao, C. Huang, and L. Chen, "Nonlinear planar secondary resonance analyses of suspended cables with thermal effects," Journal of Thermal Stresses, vol. 42, no. 12, pp. 1515-1534, 2019.

[23] Y. Zhao, H. Lin, L. Chen, and C. Wang, "Simultaneous resonances of suspended cables subjected to primary and super-harmonic excitations in thermal environments," International Journal of Structural Stability and Dynamics, vol. 19, no. 12, Article ID 1950155, 2019.

[24] Y. Zhao, C. Huang, L. Chen, and J. Peng, "Nonlinear vibration behaviors of suspended cables under two-frequency excitation with temperature effects," Journal of Sound and Vibration, vol. 416, pp. 279-294, 2018.

[25] H. Ding and J. W. Zu, "Periodic and chaotic responses of an axially accelerating viscoelastic beam under two-frequency excitations," International Journal of Applied Mechanics, vol. 5, no. 2, Article ID 1350019, 2013.

[26] I. D. Breslavsky and M. Amabili, "Nonlinear vibrations of a circular cylindrical shell with multiple internal resonances under multi-harmonic excitation," Nonlinear Dynamics, vol. 93, no. 1, pp. 53-62, 2018.

[27] H. S. Bauomy and A. T. EL-Sayed, "Vibration performance of a vertical conveyor system under two simultaneous resonances," Archive of Applied Mechanics, vol. 88, no. 8, pp. 1349-1368, 2018.

[28] X. Han, Y. Zhang, Q. Bi et al., "Two novel bursting patterns in the Duffing system with multiple-frequency slow parametric excitations," Chaos, vol. 28, Article ID 43111, 2018.

[29] F. K. Alfosail and M. I. Younis, "Multifrequency excitation of an inclined marine riser under internal resonances," Nonlinear Dynamics, vol. 99, no. 1, pp. 149-171, 2020.

[30] Y. Briend, M. Dakel, E. Chatelet, M.-A. Andrianoely, R. Dufour, and S. Baudin, "Effect of multi-frequency parametric excitations on the dynamics of on-board rotor-bearing systems," Mechanism and Machine Theory, vol. 145, Article ID 103660, 2020.

[31] M. Ghadiri, S. Hamed, and S. Hosseini, "Nonlinear dual frequency excited vibration of viscoelastic graphene sheets exposed to thermo-magnetic field," Communications in Nonlinear Science and Numerical Simulation, vol. 83, Article ID 105111, 2020.

[32] B. Kalita and S. K. Dwivedy, "Nonlinear dynamics of a parametrically excited pneumatic artificial muscle (PAM) actuator with simultaneous resonance condition," Mechanism and Machine Theory, vol. 135, pp. 281-297, 2019.

[33] H. Kang, Y. Cong, and G. Yan, "Theoretical analysis of dynamic behaviors of cable-stayed bridges excited by two harmonic forces," Nonlinear Dynamics, vol. 95, no. 3, pp. 2263-2274, 2020.

[34] T. Xu, D.-X. Hou, Z.-N. Sun, and D.-W. Guo, "Vibration characteristics of multi-parametric excitations and multifrequency external excitations of rolling mill under entry thickness fluctuation of strip," Journal of Iron and Steel Research International, vol. 27, no. 5, pp. 517-527, 2020.

[35] Q. Ren, J. Peng, and H. Chen, "Amplitude information-frequency characteristics for multi-frequency excitation of underwater active electrolocation systems," Bioinspiration and Biomimetics, vol. 15, no. 1, Article ID 16004, 2019.

[36] N. Jaber, A. Ramini, and M. I. Younis, "Multifrequency excitation of a clamped-Cclamped microbeam: analytical and experimental investigation," Microsystems and Nanoengineering, vol. 2, Article ID 16002, 2016.

[37] V. Tzanov, J. Llobet, F. Torres, F. Perez-Murano, and N. Barniol, "Multi-frequency resonance behaviour of a $\mathrm{Si}$ fractal NEMS resonator," Nanomaterials, vol. 10, no. 4, p. 811, 2020.

[38] F. Ebrahimi and S. H. S. Hosseini, "Double harmonically excited nonlinear vibration of viscoelastic piezoelectric nanoplates subjected to thermo-electro-mechanical forces," Journal of Vibration and Control, vol. 26, no. 7-8, pp. 430-446, 2020.

[39] Y. Cong, H. Kang, and G. Yan, "Investigation of dynamic behavior of a cable-stayed cantilever beam under two-frequency excitations," International Journal of Non-linear Mechanics, vol. 129, Article ID 103670, 2021.

[40] P. F. Pai, H. Peng, and S. Jiang, "Acoustic metamaterial beams based on multi-frequency vibration absorbers," International Journal of Mechanical Sciences, vol. 79, pp. 195-205, 2014.

[41] M. Rezaei, R. Talebitooti, and S. Rahmanian, "Efficient energy harvesting from nonlinear vibrations of PZT beam under simultaneous resonances," Energy, vol. 182, pp. 369-380, 2019.

[42] Y. Zheng, Z. Zhou, and H. Huang, "A multi-frequency MIMO control method for the 6DOF micro-vibration exciting system," Acta Astronautica, vol. 170, pp. 552-569, 2020.

[43] M. Sayed, A. A. Mousa, and I. Mustafa, "Stability and bifurcation analysis of a buckled beam via active control," Applied Mathematical Modelling, vol. 82, pp. 649-665, 2020.

[44] R. H. Plaut, N. Haquang, and D. T. Mook, "Simultaneous resonances in non-linear structural vibrations under twofrequency excitation," Journal of Sound and Vibration, vol. 106, no. 3, pp. 361-376, 1986. 